\section{Cost-effectiveness of preventing depression}

\section{in primary care patients}

\author{
Randomised trial \\ FILIP SMIT, GODELIEF WILLEMSE, MARC KOOPMANSCHAP, \\ SIMONE ONRUST, PIM CUIJPERS and AARTJAN BEEKMAN
}

\author{
Background Little is known about the \\ cost-effectiveness of preventing mental \\ disorders.
}

\begin{abstract}
Aims To study the cost-effectiveness of care as usual plus minimal contact psychotherapy relative to usual care alone in preventing depressive disorder.
\end{abstract}

\begin{abstract}
Method An economic evaluation was conducted alongside a randomised clinical trial. Primary care patients with subthreshold depression were assigned to minimal contact psychotherapy plus usual care $(n=107)$ or to usual care alone $(n=109)$
\end{abstract}

Results Primarycare patients with subthreshold depression benefited from minimal contact psychotherapy as it reduced the risk of developing a full-blown depressive disorder from $18 \%$ to $12 \%$. In addition, this intervention had a $70 \%$ probability of being more cost-effective than usual care alone. A sensitivity analysis indicated the robustness of these results.

Conclusions Over l year adjunctive minimal contact psychotherapy improved outcomes and generated lower costs. This intervention is therefore superior to usual care alone in terms of cost-effectiveness.

Declaration of interest None. Funding detailed in Acknowledgements.
Depression is highly prevalent, compromises the quality of life and has a substantial economic impact (Wells et al, 1992; Bijl et al, 1998; Löthgren, 2004). Several cost-effective interventions directed at depression in primary care patients are available (Schulberg et al, 2002; Scott et al, 2003; McCrone et al, 2004). However, according to one estimate the burden of depression can be averted for only $26 \%$, even under a hypothetical regimen of optimal care (Andrews et al, 2004). This leaves a formidable gap between what the best treatments can offer and the needs of many. This gap calls for interventions other than curative ones. Preventive interventions may have a role here (Smit et al, 2004, 2006). A randomised clinical trial by Willemse et al (2004) showed superior effectiveness of minimal contact psychotherapy over care as usual in preventing the onset of full-blown depressive disorder in primary care patients with sub-threshold depression. Using the same trial data, we now investigate the cost-effectiveness of this adjunctive therapy relative to usual care alone.

\section{METHOD}

The method of this trial has been described in detail by Willemse et al (2004). Here, we describe its main features and focus attention on the economic aspects.

\section{Sample}

Participants (aged 18-65 years) were recruited from 19 general practices in The Netherlands. Patients were considered to be eligible when presenting sub-threshold depression defined as having at least one core symptom plus one, two or three current depressive symptoms according to the Instel screening instrument (Tiemens et al, 1995). Exclusion criteria were the presence of full-blown DSM-IV (American Psychiatric Association, 1994) depressive disorder, dysthymia, bipolar disorder, social phobia, agoraphobia or panic disorder in the past 12 months as measured with the Composite International Diagnostic Interview (CIDI; see 'Measures').

Participants were recruited in several steps (Fig. 1). Research assistants screened 3825 patients who were waiting to see their general practitioner. Eligible patients $(n=1018)$ were asked to give their informed consent to participate in the trial. Of these, 363 were willing to do so and received a computer-assisted diagnostic interview with the CIDI. This was done to exclude patients with full-blown depression and other DSM-IV Axis I disorders as specified above. The randomisation was done centrally, using blocked randomisation stratified by general practice with the patient as unit of randomisation, with blocks of four patients. Eligible patients who had given their informed consent were randomised, with equal probability, to receive minimal contact psychotherapy adjunctive to usual care $(n=107)$ or to usual care alone $(n=109)$. Of these, 83 in the intervention group and 94 in the usual care group were retained in the trial after 12 months. Fewer participants completed the economic questionnaire: at baseline, questionnaires were completed by 99 members of the intervention group and 102 of the usual care group, of whom 75 and 87 respectively completed the questionnaires at follow-up.

The study was conducted as a pragmatic trial. Only the interviewers were unaware of the participants' randomisation status. The trial protocol was approved by an independent medical ethics committee.

\section{Intervention}

The experimental intervention was cognitive-behavioural minimal contact psychotherapy for depression, based on the Dutch version (Cuijpers, 2000) of the 'Coping with Depression' course (Lewinsohn et al, 1984). The main component was a self-help manual with instructions on mood management. The self-help therapy was guided by six short telephone calls with a prevention worker. The control intervention was care as routinely provided by the general practitioners. All participants, in both conditions, could make use of all other types of health services during the intervention period. 


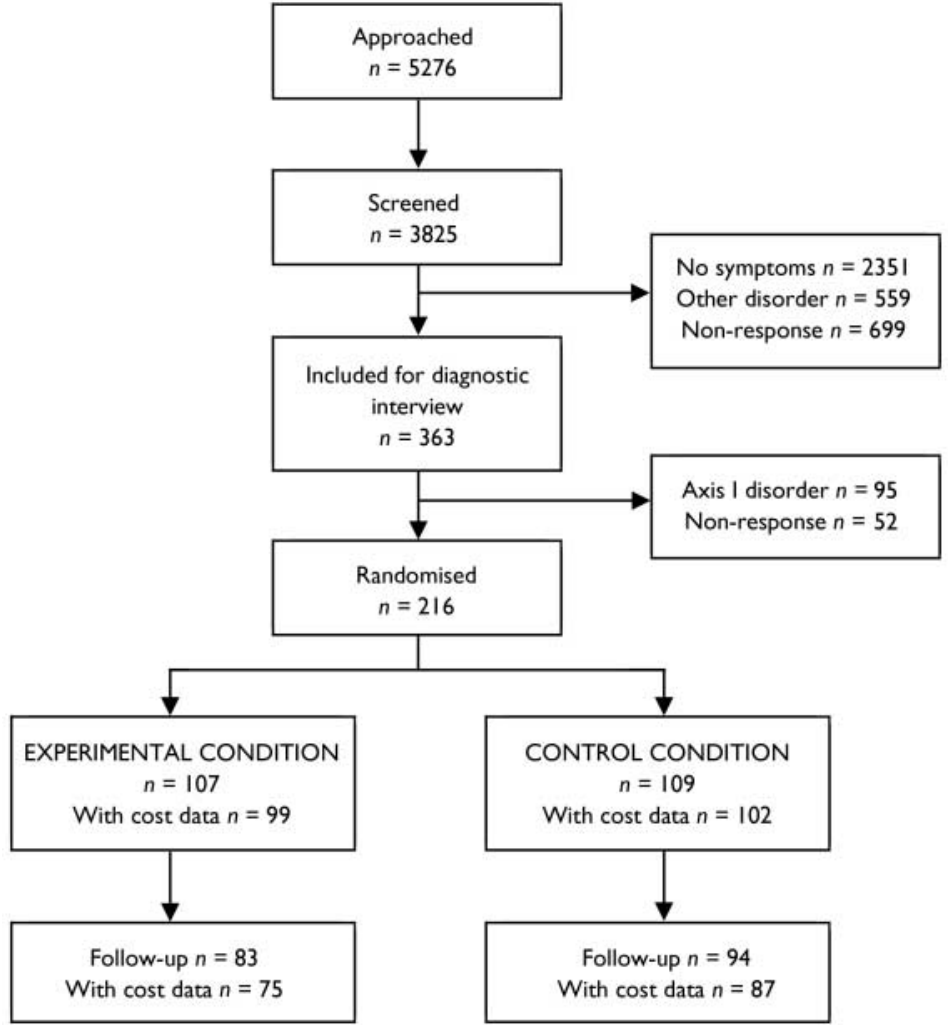

Fig. I Patient flow through study.

\section{Clinical measures}

The participants' DSM-IV depression status was assessed with the CIDI-Auto 2.1 (World Health Organization, 1997) in its Dutch version (Ter Smitten et al, 1998). The CIDI is a standardised diagnostic interview for the assessment of mental disorders, developed by the World Health Organization. It was designed for use by trained lay interviewers, has high interrater and test-retest reliability and good validity for affective and anxiety disorders (Wittchen, 1994; Andrews \& Peters, 1998). The interviews were carried out over the telephone. This should not have affected the results in any meaningful way (Rohde et al, 1997; Evans et al, 2004). Depressive symptoms were measured using the Centre for Epidemiological Studies - Depression scale (CES-D; Radloff, 1977), Dutch version (Bouma et al, 1995), a widely used selfreport scale measuring the frequency of 20 depressive symptoms during the past week. The CES-D generates a total score that can range from 0 to 60 , with a higher score indicating more depressive symptoms. The Dutch translation has good reliability and validity (Bouma et al, 1995).

\section{Measuring resource use}

For this study we adopted a societal perspective, including the costs of all types of health services and the costs that stem from production losses. The time frame of this study was restricted to 1 year. Therefore, we did not correct for inflation and did not discount costs. All costs are expressed in euros $(€)$ for the reference year 2003 on a per capita basis for the period of 1 year.

Information on the participants' use of health services was obtained with the Trimbos and Institute of Medical Technology Assessment Cost Questionnaire for Psychiatry (TIC-P; Hakkaart-van Roijen et al, 2002). With this questionnaire patients register the number of general practice visits, sessions with psychiatrists, hospital days, etc. In addition, the number of 'work loss' days (absenteeism from work) and the number of 'work cut-back' days (reduced efficiency at work while feeling ill) were also measured with help of the TIC-P.

\section{Cost of services}

The intervention costs of minimal contact psychotherapy were $€ 124$ for screening and a further $€ 124$ for the intake, plus $€ 31$ per additional contact over the telephone with a maximum of six calls. Patients had to pay $€ 25.50$ for the self-help manual. The patients' time for working through the self-help manual was valued at $€ 8.30 / \mathrm{h}$, assuming that they would carry out their assignments after office hours. It should be noted that the intervention costs occurred only in the experimental group during the actual uptake of the intervention over 4 months.

Direct medical costs are the costs of treatments offered by a broad range of both formal and informal health service providers (Table 1). Medical services were costed by multiplying the number of health service units (consultations, hospital days, etc.) by their standard cost price (Oostenbrink et al, 2002, 2004). To these we added the costs of antidepressants, calculated as the cost price per standard daily dose as reported in the Pharmaceutical Compass (http://www.fk.cvz.nl), plus $6 \%$ value added tax, multiplied by the number of prescription days, plus the pharmacist's dispensing costs of $€ 6.45$ per prescription.

Direct non-medical costs arose when patients travelled to health service providers and paid for parking. These 'out-ofpocket' costs were valued at $€ 0.16 / \mathrm{km}$ and $€ 2.50 / \mathrm{h}$ parking time. To this we added the costs of the patients' time spent in travel, waiting and in treatment at $€ 8.30 / \mathrm{h}$ (Table 1).

\section{Cost of production losses}

Indirect non-medical costs arise when production losses occur owing to illness. Three situations can be encountered here. First, people can be absent from paid work. To evaluate a lost day in a paid job we used age- and gender-specific 'friction costs' obtained from Oostenbrink et al (2004). Friction costs represent the monetary counter-value of production losses that occur during absence from work with a limit of 5 months (Koopmanschap et al, 1995). Second, production losses also occur when people are ill but continue to work with reduced efficiency. We estimated the number of work cut-back days as the number of days actually worked when ill, multiplied by a self-reported inefficiency score, which ranged between 0 and $1 \quad 0$, as efficient as when in good health; 1 , totally inefficient). Again, we used friction costs to valuate these production losses. Third, people may also be too ill to perform 
Table I Direct medical and direct non-medical costs by health service type

\begin{tabular}{|c|c|c|c|c|c|}
\hline \multirow[t]{2}{*}{ Health service type } & \multicolumn{2}{|c|}{ Direct medical costs ${ }^{\prime}$} & \multicolumn{3}{|c|}{ Direct non-medical costs ${ }^{1,2}$} \\
\hline & Unit & Cost $(€)^{3}$ & $\begin{array}{c}\text { Distance } \\
\text { travelled }(\mathbf{k m})\end{array}$ & $\begin{array}{l}\text { Patient's time } \\
\text { (h) }\end{array}$ & Cost $(€)^{4}$ \\
\hline Medical doctor & Consultation & 20.20 & 1.8 & I & 11.10 \\
\hline Medical specialist & Consultation & 98.00 & 7 & 2 & 20.20 \\
\hline Regional mental health service & Contact & 124.00 & 10 & 3 & 29.00 \\
\hline Regional addiction service $e^{5}$ & Contact & 124.00 & 10 & 3 & 29.00 \\
\hline \multicolumn{6}{|l|}{ Mental hospital } \\
\hline Out-patient & Consultation & 88.00 & 12 & 4 & 37.20 \\
\hline Day care & Contact & 125.00 & 12 & 4 & 37.20 \\
\hline In-patient & Day & 250.00 & & 8 & 66.40 \\
\hline \multicolumn{6}{|l|}{ General hospital } \\
\hline Out-patient & Consultation & 56.00 & 7 & 3 & 28.50 \\
\hline Day care & Contact & 229.00 & 7 & 4 & 36.80 \\
\hline In-patient & Day & 337.00 & & 8 & 66.40 \\
\hline \multicolumn{6}{|l|}{ Teaching hospital } \\
\hline Out-patient & Consultation & 100.00 & 12 & 3 & 29.30 \\
\hline \multicolumn{6}{|l|}{ Academic hospital } \\
\hline Day care & Contact & 229.00 & 12 & 4 & 37.60 \\
\hline In-patient & Day & 476.00 & & 8 & 66.40 \\
\hline Private practice psychotherapist & Session & 76.00 & 5 & 2 & 19.90 \\
\hline Social worker ${ }^{6}$ & Contact & 45.00 & 7 & 3 & 28.50 \\
\hline Physiotherapist & Contact & 22.75 & 1.8 & 2 & 19.40 \\
\hline Home care & Hour & 30.70 & 0 & 0 & 0.00 \\
\hline Informal care (family, friends) ${ }^{7}$ & Hour & 8.30 & 0 & 0 & 0.00 \\
\hline
\end{tabular}

I. Costs are in euros for 2003

2. Based on average distances $(\mathrm{km})$ and travel+waiting+treatment times $(\mathrm{h})$ for receiving treatment (Oostenbrink et al, 2004).

3. Integral unit cost prices (Oostenbrink et al, 2004)

4. Costs of I km $€ 0.16$, I h parking $€ 2.50$, I h patient's time $€ 8.30$ (Oostenbrink et al, 2004).

5. Valued as out-patient mental health services.

6. From DFL 77.00 in 1993, converted into euros, indexed for 2003 (cf. http://www.cbs.nl) and rounded.

7. Valued as domestic help (cf. Oostenbrink et al, 2004).

domestic tasks. These costs were evaluated at the price of domestic help at $€ 8.30 / \mathrm{h}$.

\section{Statistical analysis}

The analysis of clinical outcomes was conducted in accordance with the intentionto-treat principle. Use was made of the regression imputation procedure as implemented in Stata version 7.0 for handling loss to follow-up. In the regression imputation model, baseline CES-D scores, age and gender were used as predictors, because they were significant predictors of depression status at follow-up. Since patients were recruited from 19 general practices, some degree of clustering in the data had occurred. Clustering violates the assumption of independence of observations, and may thus affect standard errors and $P$ values. This was handled with the help of so-called robust standard errors, which were the intervention, plus the direct medical, direct non-medical and indirect costs and some of their components (see Table 2). This was done for both trial arms and for the differences between the two study groups. The corresponding tests were based on 2500 bootstrap replications because cost data are non-normally distributed.

\section{Analysis of cost-effectiveness}

In the cost-effectiveness analysis health effects (depression-free person-years) and costs in both treatment arms were computed by means of non-parametric bootstrapping (2500 times) of the individual patient data with respect to both incremental costs and incremental health effects. The comparison of the simulated differences in costs and health effects is presented in a cost-effectiveness plane (see Fig. 2), with differences in costs on the vertical axis and differences in health effects on the horizontal axis. If the intervention appears in the top left-hand quadrant of the plane, higher costs are paid for lower effectiveness; the intervention is then unacceptable from a cost-effectiveness perspective, and conventional care remains the treatment of choice. If the intervention appears in the lower right-hand quadrant, lower costs are then associated with positive health effects; the intervention dominates and is acceptable. In the other two quadrants, higher (or lower) cost levels have to be weighed against greater (or lesser) effectiveness.

A second way of illustrating the costeffectiveness results, taking into account the uncertainty, is the cost-effectiveness acceptability curve (Van Hout et al, 1994; Barrett \& Byford, 2003). Such an acceptability curve represents the probability that the intervention is cost-effective relative to usual care, given a varying threshold for the willingness to pay for a case of prevented depression (see Fig. 3). statisical test was conducted at $\alpha<0$. one-sided, because inferior effectiveness of adjunctive psychotherapy over usual care alone was not expected.

\section{Analysis of costs}

The analysis of costs was also conducted in agreement with the intention-to-treat principle. Missing cost data at follow-up were imputed as before, but now with costs at baseline, age and gender as predictors. We report the mean annual per capita costs of

\section{Sensitivity analyses}

It appeared that the total costs were dominated by the costs of production losses. Therefore, the analyses were repeated for the total costs minus those of production losses, to give an idea of the cost-effectiveness when the more narrow perspective of direct costs is used instead of the broader societal perspective. 


\section{RESULTS}

\section{Sample}

The participants were predominantly female $(66 \%)$, living with a partner $(78 \%)$ and employed $(83 \%)$. The mean age was 41 years and the participants had received 14 years of education on average. At baseline their mean CES-D score on depressive symptoms was 12.8 , well below the cutoff score of 16 above which people are considered to have clinically relevant depression. No significant difference was found between the study groups for these variables, indicating that randomisation had resulted in comparable groups (Willemse et al, 2004).

\section{Health effects}

At 12 months, the incidence rate of depressive disorder was $11.9 \%$ in the adjunctive psychotherapy group $v .18 .3 \%$ in the group receiving usual care only. The incidence rate ratio (IRR) was therefore 11.9/ $18.3=0.65$, and the 0 hypothesis of inferior clinical effects in the intervention condition had to be rejected $(\mathrm{IRR}=0.65$, s.e. $=0.15$, $t=-1.82, P=0.04$, one-sided), favouring the conclusion that adjunctive minimal contact psychotherapy is more successful than usual care alone in reducing the incidence of depressive disorder (Willemse et al, 2004).

\section{Costs}

Over 1 year the adjunctive psychotherapy group incurred the costs of the intervention (on average €423), but these additional costs were partly compensated for by savings elsewhere in the medical sector (Table 2): the mean difference of the direct medical costs was $€ 60$ (s.e. $=555$ ) in favour of care as usual, but this was not statistically significant $(P=0.914)$. Moreover, the out-of-pocket costs of the patients in the intervention condition were somewhat lower (€441 v. $€ 507$ in the usual care condition), representing a difference of $€ 66$ in favour of the intervention. Avoiding production losses resulted in further cost savings. The mean cost of the production losses was $€ 4638$ in the intervention condition against a higher $€ 6481$ in the usual care condition, resulting in an average saving of $€ 1843$. Overall, the mean annual per capita total cost in the intervention group was $€ 6766$, which compares favourably with the $€ 8614$ in the usual care group. The overall savings average $€ 1849$ when the psychotherapy

Table 2 Annual per capita costs categorised by condition

\begin{tabular}{|c|c|c|c|c|c|c|c|}
\hline & \multicolumn{7}{|c|}{ Annual per capita cost $(€)$} \\
\hline & \multicolumn{2}{|c|}{ Experimental group $(E)$} & \multicolumn{2}{|c|}{ Control group $(C)$} & \multicolumn{3}{|c|}{ Difference $(E-C)$} \\
\hline & Mean & (s.e.) & Mean & (s.e.) & Diff. & (s.e.) & $P$ \\
\hline Direct medical costs & 1687 & $(305)$ & 1627 & (419) & 60 & $(555)$ & 0.914 \\
\hline Intervention & 423 & (13) & 0 & (0) & 423 & $(12)$ & 0.000 \\
\hline GP & 165 & (30) & 152 & (14) & 13 & (32) & 0.681 \\
\hline Antidepressants & 27 & (9) & 29 & $(10)$ & -2 & (7) & 0.789 \\
\hline Other medical & 1067 & (291) & 1442 & $(431)$ & -376 & $(52 I)$ & 0.471 \\
\hline Direct non-medical costs & 441 & (59) & 507 & (77) & -66 & (88) & 0.453 \\
\hline Indirect non-medical costs & 4638 & $(1634)$ & 6481 & $(1393)$ & -1843 & (1639) & 0.261 \\
\hline Work loss & 2374 & (807) & 3279 & $(697)$ & -905 & $(833)$ & 0.277 \\
\hline Work cut-back & 2232 & $(823)$ & 3175 & $(696)$ & -942 & (796) & 0.237 \\
\hline Domestic & 31 & (I3) & 28 & (9) & 4 & (I7) & 0.828 \\
\hline Total cost & 6766 & $(17 \mid 2)$ & 8614 & $(1490)$ & -1849 & $(I 7 \mid 5)$ & 0.281 \\
\hline
\end{tabular}

intervention is added to care as usual, but this is statistically not significant $(95 \% \mathrm{CI}$ -5169 to $1472 ; P=0.281)$. Nevertheless, it is worth noting that there is a large probability that the costs of the intervention are balanced by savings elsewhere. We return to this point shortly.

\section{Cost-effectiveness}

The incremental cost-effectiveness ratio was calculated as $\left(C_{1}-C_{0}\right) /\left(E_{1}-E_{0}\right)$, where $C$ is the average annual per capita cost and $E$ is the percentage of people who did not develop depression in the experimental and control conditions (subscripted 1 and 0 respectively). In other words, the incremental cost-effectiveness ratio is the difference of mean costs between the

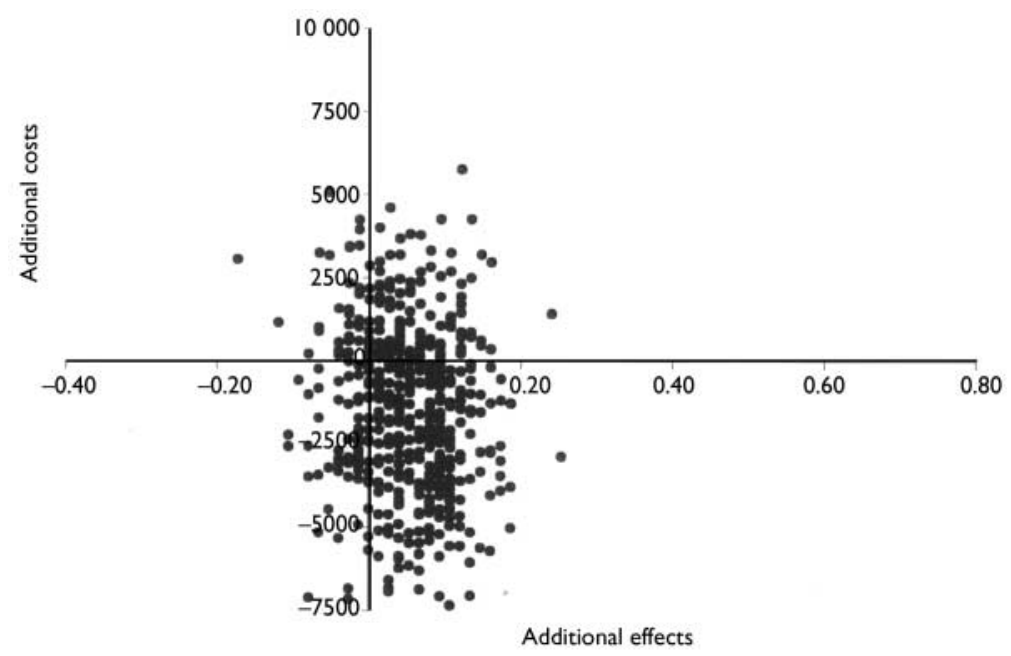

Fig. 2 Cost-effectiveness plane: each dot $(n=2500)$ represents a bootstrapped cost-effectiveness ratio. conditions divided by the difference in effect. Substitution yields $(6766-8614) /$ $(88.1-81.7)=-288.75$. Hence, for each case of depression that can be avoided by offering the experimental treatment instead of care as usual, a saving is made of $€ 288.75$.

The incremental cost-effectiveness ratio is surrounded by a certain amount of uncertainty. Figure 2 presents the costeffectiveness plane for the intervention $v$. care as usual. The incremental costs are plotted on the $y$ axis and the incremental effects on the $x$ axis. Each dot $(n=2500)$ represents a bootstrap replication of the incremental cost-effectiveness ratio; $59 \%$ of the dots are in the lower right-hand quadrant, indicating a 59\% probability that minimal contact psychotherapy is the 


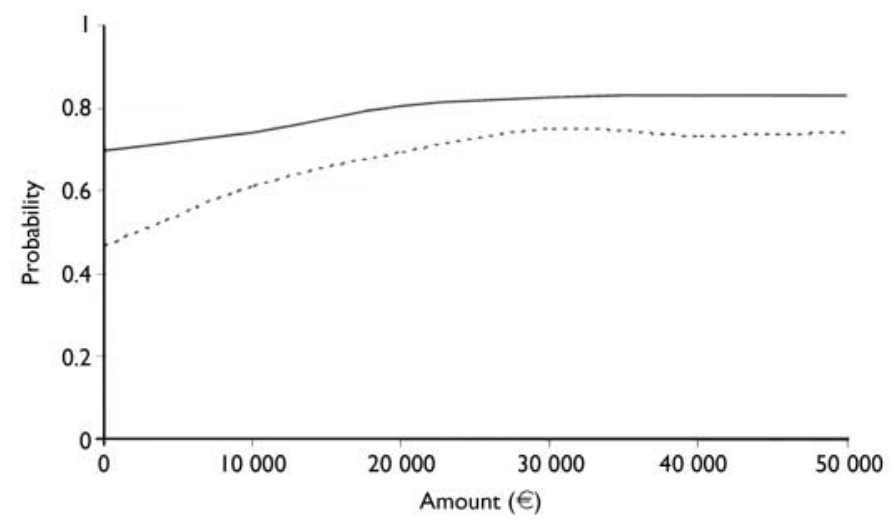

Fig. 3 Cost-effectiveness acceptability curve: probability that the incremental cost-effectiveness ratio is acceptable ( $y$-axis) given varying thresholds for willingness to pay ( $x$-axis) for total costs (solid line) and direct costs (dotted line), based on 2500 bootstrap replications.

superior treatment, because it generates better health effects against lower costs when compared with care as usual. On the other hand, there is a $5 \%$ probability that this psychotherapy is inferior, and there is a $10 \%$ probability that it is both less costly and less effective. A fifth (21\%) of the dots fall in the upper right-hand quadrant, indicating that a health gain is produced, but at additional costs.

\section{Acceptability}

Figure 3 presents the cost-effectiveness acceptability curve for minimal contact psychotherapy $v$. care as usual. The solid line curve intersects the $y$ axis at 0.70 ; when the willingness to pay for an averted depressive episode is absent (equal to $€ 0.00$ ), then there is a $70 \%$ probability that this therapy is more cost-effective than care as usual. Generally, people are willing to pay for avoiding a depressive episode, and minimal contact psychotherapy will be regarded as good value for money given a - usually unknown - ceiling for this willingness to pay. Different ceilings are presented on the $x$ axis. When the willingness to pay is raised to $€ 10000$ per avoided depression, then the intervention has a probability of $74 \%$ of being cost-effective compared with its alternative; at $€ 20000$ the probability of an acceptable cost-effectiveness has risen to $80 \%$ and at $€ 30000$ it has reached a $83 \%$ probability of being more acceptable than usual care alone.

\section{Sensitivity analysis}

When the indirect costs related to the production losses are excluded, then the distribution of the bootstrapped costeffectiveness ratios over the cost-effectiveness plane is as follows:

(a) $41 \%$ of the ratios fall in the upper righthand quadrant, indicating that better effects are obtained against higher costs;

(b) $11 \%$ fall in the upper left-hand quadrant, indicating that the intervention is inferior;

(c) $7 \%$ fall in the lower left-hand quadrant, indicating that the intervention has worse clinical outcomes against lower costs;

(d) $39 \%$ of the bootstrapped cost-effectiveness ratios fall in the lower right-hand quadrant, implying that the intervention is superior, because it generates better outcomes against lower costs than care as usual.

Under these circumstances, the psychotherapy intervention has a probability of $46 \%$ of being acceptable when the willingness to pay equals 0 (dotted line in Fig. 3). When the willingness to pay is increased to $€ 10000, € 20000$ and $€ 30000$, then the probability of the intervention being more acceptable than usual care increases to $61 \%, 70 \%$ and $75 \%$ respectively.

\section{DISCUSSION}

\section{Main findings}

The incidence of DSM-IV Axis I depression among participants in our study's control condition was $18 \%$ at the end of the intervention period. The incidence was significantly lower in those assigned to the experimental condition, at $12 \%$ (Willemse et al, 2004). This represents a reduction in the incidence by one-third, and indicates superior effectiveness of adjunctive minimal contact psychotherapy compared with care as usual. Not only is the intervention more effective, this economic evaluation indicates that choosing it over usual care alone is likely to be the best treatment option, because there is a $70 \%$ probability that the intervention is preferable to usual care when the costs of production losses are included in the analysis. Excluding the latter costs results in a comparable situation. From the cost-effectiveness perspective, this intervention then has a $46 \%$ probability of being more acceptable than usual care. When the willingness to pay for avoiding a depressive episode is $€ 30000$, then the intervention has a $75 \%$ probability of being the preferred option.

\section{Limitations}

This study has several limitations. First, although problems with attrition are common in randomised trials of psychological interventions in general practice (Muñoz et al, 1995; Fairhurst \& Dowrick, 1996), the representativeness of the sample can be questioned. In recognition of this limitation, all analyses were conducted in accordance with the intention-to-treat principle, and imputation was used as a means of overcoming the missing data problem resulting from loss to follow-up. Second, it was not possible to mask participants to the condition to which they were assigned. This is true for most randomised trials of psychological interventions, but it may nevertheless have distorted the outcomes of our trial. Third, the study was conducted in The Netherlands, and the results cannot be reliably generalised to countries that have different primary care systems. Fourth, the costs and effects were considered in the time-span of 1 year. We do not know how the cost-effectiveness of minimal contact psychotherapy is affected when a longer period is used. Because of these limitations, the results of this study should be considered with some caution.

\section{The wider context}

Depression is one of the leading causes of disability (Ustun et al, 2004). However, according to one estimate, even under a hypothetical regimen of optimal (evidencebased) care the burden of depression can only be averted for about a quarter of patients (Andrews et al, 2004). This suggests 
that prevention may have to play a more important role in public mental health (Willemse et al, 2004). Reducing the burden of depression by means of a preventive intervention is possible, as was shown in a randomised clinical trial by Willemse et al (2004) and in a metaanalysis of randomised prevention trials by Cuijpers et al (2005). Perhaps of equal importance is the opportunity to offer a low-cost, self-help treatment, which is effective but consumes small amounts of healthcare resources. In fact, our study showed that there is a $70 \%$ probability that minimal contact psychotherapy as an adjunct to usual care is more cost-effective than usual care alone. Our findings are in agreement with the reviews of both Churchill et al (2001) and Schulberg et al (2002) and the more recent cost-effectiveness analysis of McCrone et al (2004). They found that psychological interventions based on cognitive-behavioural therapy are cost-effective in primary care patients with depression. Cognitive-behavioural therapy appears also to be cost-effective for relapse prevention in chronic depression (Scott et al, 2003). In contrast, other types of psychological interventions - specifically (psychodynamic) counselling and sometimes interpersonal therapy-have not shown similar effects and cost-effectiveness (Lave et al, 1998; Bower et al, 2000; Simpson et al, 2003). Now, our study adds the information that a self-help intervention based on cognitive-behavioural therapy with minimal guidance is cost-effective in avoiding the onset of full-blown depressive disorder in primary care patients with subclinical depression.

\section{Directions for the future}

The 'Coping with Depression' course (Lewinsohn et al, 1984) and its Dutch version (Cuijpers, 2000) can be used as a cost-effective adjunct to conventional primary care in order to reduce the incidence of depressive disorder. This choice is likely to result in health gains and economic benefits. Therefore, its dissemination seems appropriate. Two issues need more in-depth exploration. First, we need to know more about the cost-effectiveness of this therapy in the long run. Second, the course should perhaps be adapted for use over the internet; this might help to reduce the costs of providing this therapy, and at the same time promote its use by a larger segment of the population.

\section{CLINICAL IMPLICATIONS}

- Minimal contact psychotherapy reduces the risk of developing a full-blown depressive disorder by $30 \%$ in primary care patients with sub-threshold depression.

- Adjunctive minimal contact psychotherapy has a $70 \%$ probability of being more cost-effective than care as usual alone.

- These findings appear to be robust when excluding the costs of production losses.

\section{LIMITATIONS}

Cost and benefits are considered in the relatively short time frame of I year.

Participants could not be masked to the interventions.

The findings may not be generalisable to other countries.

FILIP SMIT, MSc, Trimbos Institute (Netherlands Institute of Mental Health and Addiction), Utrecht, and Department of Clinical Psychology, Free University, Amsterdam; GODELIEF WILLEMSE, PhD, Trimbos Institute, Utrecht; MARC KOOPMANSCHAP, PhD, Institute of Medical Technology Assessment, Erasmus Medical Centre, Rotterdam; SIMONE ONRUST, MSc, Trimbos Institute, Utrecht; PIM CUIJPERS, PhD, Department of Clinical Psychology, Free University, Amsterdam, and Trimbos Institute, Utrecht; AARTJAN BEEKMAN, MD, PhD, Department of Psychiatric Epidemiology and Department of Psychiatry, Free University, Amsterdam, and Trimbos Institute, Utrecht, The Netherlands

Correspondence: Filip Smit, Department of Prevention and Early Intervention, Trimbos Institute, PO Box 725, 2500 AS Utrecht, The Netherlands. Tel: +31 30295 9254; fax: +31 30297 IIII ; e-mail: fsmit@trimbos.nl

(First received 5 November 2004, final revision 28 June 2005, accepted I2 July 2005)

\section{ACKNOWLEDGEMENTS}

We thank all the patients and general practitioners who took part in this trial. We also thank the preven tion specialists and clinicians from the community mental health centres for guiding patients through the minimal contact intervention. The clinical trial was financially supported by the Healthcare Re search Council of the Netherlands (ZonMw), grant 22000026/97-2-268). The economic evaluation was financially supported by ZonMw (grant 2010 0964-3I) and the Ministry of Public Health, Welfare and Sports (grant 63-505). We thank Frans Rutten and Jan Oostenbrink of the Institute of Medical Technology Assessment and three anonymous referees for their advice.

\section{REFERENCES}

American Psychiatric Association (1994) Diagnostic and Statistical Manual of Mental Disorders (4th edn) (DSM-IV). Washington, DC: APA.

\section{Andrews, G. \& Peters, L. (1998) The psychometric} properties of the Composite International Diagnostic Interview. Social Psychiatry and Psychiatric Epidemiology, 33, 80-88.

Andrews, G., Issakidis, C., Sanderson, K., et al (2004) Utilising survey data to inform public policy: comparison of the cost-effectiveness of treatment of ten mental disorders. British Journal of Psychiatry, 184, 526-533.

Barrett, B. \& Byford, S. (2003) Acceptable mental health care? A new decision-making tool for costeffectiveness analysis. Mental Health Research Review, 9 $39-4 \mid$.

Bijl, R.V., Ravelli, A., Van Zessen, G. (1998) Prevalence of psychiatric disorder in the general population: results of the Netherlands Mental Health Survey and Incidence Study (Nemesis). Social Psychiatry and Psychiatric Epidemiology, 33, 587-595.

Bouma, J., Ranchor, A. V., Sanderman, R., et al (1995) Assessment of Depressive Symptoms with the CES D: Manual (in Dutch). Groningen: Noordelijk Centrum voor Gezondheidsvraagstukken/Rijksuniversiteit Groningen.

Bower, P., Byford, S., Sibbald, B., et al (2000) Randomised controlled trial of non-directive counselling, cognitive-behaviour therapy, and usual general practitioner care for patients with depression II: cost effectiveness. BMJ, 32I, 1389-1392.

Cuijpers, P. (2000) The Coping with Depression Course (in Dutch). Utrecht/Baarn: Trimbos Institute/Intro.

Cuijpers, P., Van Straten, A. \& Smit, F. (2005) Preventing the incidence of new cases of mental disorders: a meta-analytic review. Journal of Nervous and Mental Disease, 193, 119-125.

Churchill, R., Hunot, V., Corney, R., et al (200I) A systematic review of controlled trials of the effectiveness 
and cost-effectiveness of brief psychological treatments for depression. Health Technology Assessment, 5, I-173.

\section{Evans, M., Kessler, D., Lewis, G., et al (2004)}

Assessing mental health in primary care research using standardized scales: can it be carried out over the telephone? Psychological Medicine, 34, 157-162.

Fairhurst, K. \& Dowrick, C. (1996) Problems with recruitment in a randomised controlled trial of counselling in general practice: causes and implications. Journal of Health Services and Research Policy, I, 77-80.

Hakkaart-Van Roijen, L., Van Straten, A., Donker, M., et al (2002) Manual: Trimbos/iMTA Questionnaire for Costs Associated with Psychiatric Illness (in Dutch). Rotterdam: Erasmus University.

Koopmanschap, M. A., Rutten, F. F. H., Van Ineveld, B. M., et al (1995) The friction cost method for estimating the indirect costs of disease. Journal of Health Economics, 14, 17|-189.

Lave, J. R., Frank, R. G., Schulberg, H. C., et al (1998) Cost-effectiveness of treatments for major depression in primary care practice. Archives of General Psychiatry, $\mathbf{5 5}$ 645-651.

Lewinsohn, P. M., Antonucci, D. O., Breckenridge, J. S., et al (1984) The Coping with Depression Course. Eugene: Castalia.

Löthgren, M. (2004) Economic evidence in affective disorders: a review. European Journal of Health Economics, 5 (suppl. I), SI2-SI9.

McCrone, P., Knapp, M., Proudfoot, J., et al (2004) Cost-effectiveness of computerised cognitivebehavioural therapy for anxiety and depression in primary care: randomised controlled trial. British Journal of Psychiatry, 185, 55-62.

Muñoz, R. F., Ying, Y., Bernal, G., et al (1995)

Prevention of depression with primary care patients: a randomized controlled trial. American Journal of Community Psychology, 23, 199-222.

Oostenbrink, J. B., Koopmanschap, M. A. \& Rutten, F. F. H. (2002) Standardisation of costs: the Dutch manual for costing in economic evaluations.

Pharmacoeconomics, 20, 443-454.

Oostenbrink, J. B., Bouwmans, C. A. M., Koopmanschap, M. A., et al (2004) Manual for Costing: Methods and Standard Costs for Economic Evaluations in Health Care (in Dutch). Diemen: Health Insurance Board.

Radloff, L. S. (1977) The CES-D scale: a self-report depression scale for research in the general population. Applied Psychological Measurement, I, 385-40I.

Rohde, P., Lewinsohn, P. M. \& Seely, J. S. (1997) Comparability of telephone and face-to-face interviews in assessing axis I and II disorders. American Journal of Psychiatry, 154, I593-1598.

Schulberg, H. C., Raue, P. J. \& Rollman, B. L. (2002) The effectiveness of psychotherapy in treating depressive disorders in primary care: clinical and cost perspectives. General Hospital Psychiatry, 24, 203-212.

Scott, J., Palmer, S., Paykel, E., et al (2003) Use of cognitive therapy for relapse prevention in chronic depression: cost-effectiveness study. British Journal of Psychiatry, 182, 221-227.

Simpson, S., Corney, P., Fitzgerald, P., et al (2003) A randomized controlled trial to evaluate the effectiveness and cost-effectiveness of psychodynamic counselling for general practice patients with chronic depression. Psychological Medicine, 33, 229-239.

Smit, F., Beekman, A., Cuijpers, P., et al (2004) Selecting key-variables for depression prevention: results from a prospective population-based cohort study. Journal of Affective Disorders, 8I, 24I-249.
Smit, F., Ederveen, A., Cuijpers, P., et al (2006) Opportunities for cost-effective prevention of late-life depression: an epidemiological approach. Archives of General Psychiatry, in press.

Ter Smitten, M. H., Smeets, R. M. W. \& Van den Brink, W. (1998) Composite International Diagnostic Interview (CIDI), version 2.I, 12-months (in Dutch). Amsterdam/Geneva: World Health Organization.

Tiemens, B. G., Ormel, J., van den Brink, R. H. S., et al (1995) Detecting depression and generalised anxiety disorder in the primary care setting: development of a screener (in Dutch). Tijdschrift voor Sociale Gezondheidszorg, 73, 520-527.

Üstün, T. B., Ayuso-Mateos, J. L., Chatterji, S., et al (2004) Global burden of depressive disorders in the year 2000. British Journal of Psychiatry, 184, 386-392.

Van Hout, B. A., Maiwenn, J. A., Gilad, S. G., et al (1994) Costs, effects and C/E-ratios alongside a clinical trial. Health Economics, 3, 309-319.

Wells, K. B., Burnam, A., Rogers, W., et al (1992) The course of depression in adult outpatients. Archives of General Psychiatry, 49, 788-794.

Willemse, G. R.W. M., Smit, F., Cuijpers, P., et al (2004) Minimal-contact psychotherapy for subthreshold depression in primary care: randomised trial. British Journal of Psychiatry, 185, 416-421.

Wittchen, H.-U. (1994) Reliability and validity studies of the WHO-Composite International Diagnostic Interview (CIDI): a critical review. Journal of Psychiatric Research, 28, 57-84.

World Health Organization (1997) Composite International Diagnostic Interview (CIDI), version 2.I, 12-months. Geneva: WHO 